\title{
Nasotransmucosal Delivery of Curcumin-Loaded Mucoadhesive Microemulsions for Treating Inflammation-Related CNS Disorders
}

\author{
(1) Mukeshkumar Shamalbhai PATEL ${ }^{1 *}$, (1) Snigdha Das MANDAL², (1) Surjyanarayan MANDAL ${ }^{3}$, (1) Shital FALDU4 ${ }^{4}$, (1) Jayvadan PATEL ${ }^{5}$ \\ ${ }^{1}$ Wockhardt USA LLC/Morton Grove Pharmaceuticals, Inc., Senior Process Engineer, Morton Grove, United States of America \\ 2Parul University, Department of Pharmacy, Gujarat, India \\ ${ }^{3}$ AGIO Pharmaceuticals Ltd., Gujarat, India \\ 4 Smt. R.D. Gardi B. Pharmacy College, Gujarat, India \\ 5Sankalchand Patel University, Faculty of Pharmacy, Department of Pharmacy, Gujarat, India
}

\begin{abstract}
Objectives: This investigation was aimed at designing an effective mucoadhesive microemulsion system to accomplish higher brain uptake of curcumin through intranasal route.

Materials and Methods: Mucoadhesive microemulsion of curcumin (MMEC) was developed using screened oil, surfactant, and co-surfactant by Box-Behnken design and was evaluated for mucoadhesion, stability, and naso-ciliotoxicity study. Comparative brain uptake of curcumin after nasal administration of MMEC and polycarbophil curcumin gel and intravenous administration of plain curcumin solution was studied by performing biodistribution study in Swiss albino rats.

Results: The results showed that all formulation variables i.e., the amount of capmul MCM (X1), $S_{\text {mix }}$ (accenon CC: transcutol P) (X2) and percentage of aqueous. Polycarbophil $(X 3)$ had a significant effect $(p<0.05)$ on the responses. The developed MMEC was stable and non-ciliotoxic with 66.74 $\pm 3.46 \mathrm{~nm}$ and $98.58 \% \pm 1.21$ as average globule size and drug content, respectively. Polydispersibility index $(0.133 \pm 0.17)$ data and transmission electron microscopy study depicted the narrow size distribution of MMEC. Furthermore, following a comparative investigation of the brain uptake of curcumin among MMEC, plain drug gel and intravenous administration at $2.86 \mathrm{mg} / \mathrm{kg}$, more brain uptake of curcumin was demonstrated for MMEC over intravenous application. Moreover, curcumin uptake in olfactory bulb after nasal administration of MMEC (31.11 \pm 1.6$)$ was than 9.44 times higher than intravenous injection of curcumin solution ( $3.25 \pm 1.01)$. Area under curve represents the ratio of $2.86 \mathrm{mg} / \mathrm{kg}$ in brain tissue to plasma acquired afterward(s) the intranasal injection of MMEC (and it) was essentially greater than after the intravenous administration of curcumin solution.

Conclusion: Findings of the investigation revealed that optimal MMEC and intranasal route may be considered to be promising and an alternative approach for brain targeting of curcumin.
\end{abstract}

Key words: Intranasal delivery, microemulsion, brain-targeting, MMEC, mucoadhesion, TEM, curcumin

\section{INTRODUCTION}

Brain targeting through the nasal route has become a fruitful research platform, and in recent times, it is attracting a lot of attention around the globe. Drug delivery through the nasal route offers numerous benefits like quick bioavailability of the drug, circumvention of liver first-pass metabolic rate and better-delivering drug to the brain through the olfactory region (OR).' The olfactory part of the nasal route has a large surface area, i.e., $10 \mathrm{~cm}^{2}$ and acts as a relatively more vital region of drug-carrying to the central nervous system (CNS) and cerebrospinal fluid. The connective tissue of the olfactory area is known as lamina propria that comprises blood vessels and axons. ${ }^{2}$ Several studies have reported literature the brain target drugs insoluble in water via nasal route. ${ }^{3}$ However, the intranasal administration of drug is connected to numerous intrinsic problems. The fundamental problem is its adequate 
nasal volume in humans, which is $\leq 400 \mu \mathrm{L}$ ( $200 \mu \mathrm{L}$ per nose), that is undeniably a challenge for the formulators to develop a suitable formulation for poorly water-soluble drugs. ${ }^{4}$ Secondly, the rapid proliferation of the nasal mucosal layer (every 10 to $15 \mathrm{~min}$ ) and ciliary movement facilitate the drug molecules clear from the nasal cavity. ${ }^{2}$ Naso-ciliary clearance as a natural defense mechanism instead decreases the adhesion of the preparation to the nasal mucosa over a longer period. ${ }^{5}$

Curcumin is a bioactive constituent present in the rhizomes of Curcuma longa $\mathrm{L}$. Curcumin exhibits pharmacologic effects such as anti-inflammatory, anticancer, antibacterial, antirheumatic, antimalarial, antioxidant, cardioprotective, nephroprotective, neuroprotective, and hepatoprotective effects. ${ }^{6}$ But curcumin is a phytoconstituent, which is absorbed, when given through the oral route leading to very poor oral bioavailability (less than $4 \%$ ), which in fact is due to its poor aqueous solubility and erratic dissolution. ${ }^{2,3}$ Further, its incomplete oral absorption and high first-pass metabolism make it difficult to enter the brain due to $P$-glycoprotein overexpression limit its clinical usefulness. ${ }^{7-9}$ Therefore, we have tried herein to improve the brain targeting of curcumin by developing a new formulation through the nasal route of administration.

A microemulsion (ME) is an anisotropically precise and thermodynamic stable liquid formulation with a globule size $<200 \mathrm{~nm}$. It is composed of oil and water phases, stabilized by a concrete mixture of surfactant and co-surfactant $\left(\mathrm{S}_{\text {mix }}\right)^{10-}$ ${ }^{14}$ Several studies in the literature revealed the application of ME for intranasal, topical, and parenteral, transdermal, and oral drug delivery systems. ${ }^{7,15}$ Additionally, o/w ME is a better choice for drug incorporation with low water solubility due to its solution-like feature, which provides uniform of dose. But, the ME used for intranasal administration becomes the future area for the CNS targetting. ${ }^{16,17}$ The addition of a suitable mucosal adhesive (polycarbophil) such as a polyelectrolyte polymer helps overcome the difficulties associated with the nasal route of drug delivery by retaining the ME formulation on the nasal mucosa for a more extended period. The tissue appropriation and blood-cerebrum (CRB) obstruction entrance information. The kinetics of tissue distribution and blood-brain barrier penetration data revealed that curcumin and nanoformulation were efficient enough for brain targeting.,17,18 Moreover, used a quality by design approach was to develop a suitable ME formulation for intranasal delivery, which ultimately maximizes brain targeting. ${ }^{2-4,16-20}$

Therefore, this research aimed to develop an optimal mucoadhesive microemulsion system (MMEC) and perform a comparative brain distribution study of curcumin following intranasal and intravenous administration in the rat. It is thought that MMECs can effectively distribute curcumin in the brain due to the unique connection between the brain and the nose, and the controlled release capability of the developed formulation. ${ }^{2-4,18-20}$ Thus, it can provide an innovative method for treating inflammatory diseases of the CNS.

It is thought that MMEC might be effective enough to distribute curcumin within and into the brain due to the unique nose- brain connection and the controlled release capability of the formulation..$^{2-4}$ It can provide an innovative approach for treating inflammation-related CNS disorders.

\section{MATERIALS AND METHODS}

Curcumin and emodin were acquired from Arjuna Natural Pvt. Ltd. (Kerala, India). Capex, Capmul MCM, Cremophor EL, accenon $\mathrm{CC}$, and transcutol $\mathrm{P}$ was obtained from ABITEC Corporation (Columbus, USA). Polycarbophil was received from Lubrizol India Pvt. Ltd., (Navi Mumbai, India). Labral M 1944CS and labrafac CC were acquired from Gattefosse, Navi Mumbai, isopropyl myristate, PEG 400, tween-80, PEG 600, glycerol, isopropyl alcohol, tween-60, oleic acid, and isobutyl alcohol, were procured from Sigma-Aldrich (Bangalore, India). Propranolol was obtained from Torrent Pvt. Ltd. (Ahmedabad, India). Hexane, diethyl ether, $\mathrm{NaH}_{2} \mathrm{PO}_{4}, \mathrm{Na}_{2} \mathrm{HPO}_{4}$, $p$-phenyl phenol, and acetonitrile [high performance liquid chromatography (HPLC) grade] were acquired from Merck Life Science Pvt (Bengaluru, India). All the reagents used were of analytical grade. Double distilled pure water was used in the present research.

\section{Experimental method}

\section{Animals}

All animal experiments were performed using (National Institute of Health instructions for the care and use of laboratory animals publishing \# 85-23, revised 1996). The Institutional Animal Ethical Committee approved the animal experiment (CPCSEA no: 984/14/11/CPCSEA) (New Delhi, India). Albino rats (230-270 g) were obtained from Zydus Cadila Healthcare Ltd., Moriya, and Ahmedabad, India. The rats were fasted for approximately 12-18 hours with free access to water and quality food through research. The rats were maintained at constant room temperature $\left(25 \pm 2^{\circ} \mathrm{C}\right)$ and air humidity $(50 \pm 10 \%)$ with a light/dark cycle of $12 \mathrm{~h}$.

\section{Pre-formulation study}

The selection of oil as internal phase and core phases for intranasal o/w MME was mainly made on drug solubility in nasomucosal compatible oils. ${ }^{3}$ The solubility of the drug in various screened oils such as labrafil M 1944CS, isopropyl myristate, capmul MCM, labrafac CC, and oleic acid for the intranasal drug delivery was determined through saturation solubility technique. Surfactants such as tween-60, tween-80, captex-355, accenon CC, and cremophor $\mathrm{RH} 40$ with hydrophiliclipophilic balance (HLB) values ranging between 12 and 16, were screened for curcumin solubility. Screening of co-surfactants was based on their ability to form stable and transparent MEs at a minimum concentration, and few reported co-surfactants like PEG 400, PEG 600, propylene glycol, glycerol, isobutyl alcohol, isopropyl alcohol, and transcutol $P$ were screened.,721 Excess of curcumin was added to each cap vial containing $5 \mathrm{~mL}$ of each selected vehicle. After sealing, mixtures were shaken with a shaker at $37^{\circ} \mathrm{C}$ for $24 \mathrm{~h}$. After reaching equilibrium, centrifuged each vial was kept at 8000 rpm for $15 \mathrm{~min}$, and excess insoluble curcumin was separated by filtering the supernatant by 
Whatman filter $(0.45 \mu \mathrm{m})$. Solubilized drug concentration from the supernatant was quantified by ultraviolet-visible (UV-VIS) spectrophotometer (Shimadzu UV-1800) 2,5

\section{Preparation of MMEC}

Amount of oil and surfactant-cosurfactant ratio $\left(\mathrm{S}_{\text {mix }}\right)$ was selected from pseudo-ternary phase diagram data. MME was then prepared by water titration method using the screened formulation compositions and was optimized by Box-Behnken design of Design-Expert ${ }^{\circledR}$ software (Stat-Ease, Inc., Minneapolis, Minnesota, USA, V 7.1.0). 7,11 Three independent factors such as percentage amount of capmul MCM (X1), $\mathrm{S}_{\text {mix }}$ (accenon CC: transcutol $P, X 2)$, and percent measure of polycarbophil (X3, as far as \% w/v in water) with their three levels. It is taken from preliminary experiments. ${ }^{8} \mathrm{~A}$ total of 15 formulation compositions of MMEC were obtained. The polynomial model and equation were experimentally interpreted based on significant terms $(p<0.05)$ and non-significant lack of fit data as provided by Design-Expert ${ }^{\circledR}$ software to define the influence of independent variables on the responses. ${ }^{22,23}$ The experimental design was quadratic and details of the three levels taken are demonstrated in Table 1. MMECs were prepared experimentally using the compositions of all model MMECs as summarized in Table 1. $\mathrm{S}_{\text {mix }}$ i.e., a mixture of accenon CC and transcutol P (3:1), was mixed well with drug-dissolved capmul MCM solution. The above mixture was then titrated with different aqueous polycarbophil concentrations with gentle and continuous stirring at room temperature using a magnetic stirrer..$^{10,21,23}$ Finally, it was decided on standard droplet size, flux, retention time, and drug release (\%) to be measured experimentally for all fifteen batches. Flux was estimated from the ex vivo permeation study. Plain curcumin dispersed polycarbophil gel [polycarbophil curcumin gel (PCG) $3.0 \mathrm{mg} / \mathrm{mL}$ ] was prepared by dispersing $30 \mathrm{mg}$ curcumin to the already prepared $0.5 \%$ aqueous-based plain polycarbophilbased gel with continuous stirring.

\section{Optimization of MMEC}

Responses like average globule size, flux, mucoadhesive potential, and drug release (\%) were selected for numerical and graphical optimization. It was decided to choose the maximum flux and drug release (\%), while having a minimum average globule size with suitable mucoadhesive potential to obtain an optimized formulation. From the overlay plot, the best composition as MMEC was visually selected. Finally, for verification, checkpoint batches were prepared experimentally and predicted values of all four responses were compared with the observed value. The best-suited composition was considered an optimized batch and was used for further study.

Table 1. Details of variables with levels, compositions and responses of MMEC model formulations provided by Box-Behnken design

\begin{tabular}{|c|c|c|c|c|c|c|c|}
\hline Batch & $\mathrm{X}_{1}$ & $X_{2}$ & $\mathrm{X}_{3}$ & Globule size $(\mathrm{nm})$ & Flux $\left(\mu \mathrm{g} / \mathrm{cm}^{2} \mathrm{~h}\right)$ & $\mathrm{RT}(\min )$ & Drug release (\%) \\
\hline $\mathrm{F} 1$ & 0.10 & 1.55 & 0.50 & 59.62 & 13.8 & 17.6 & 96.1 \\
\hline $\mathrm{F} 2$ & 0.50 & 1.55 & 0.50 & 76.36 & 18.3 & 21.5 & 83.1 \\
\hline F3 & 0.10 & 1.65 & 0.50 & 55.82 & 16.3 & 23.4 & 80.0 \\
\hline $\mathrm{F} 4$ & 0.50 & 1.65 & 0.50 & 62.59 & 14.2 & 22.1 & 86.2 \\
\hline F5 & 0.10 & 1.60 & 0.25 & 56.44 & 15.4 & 16.2 & 90.4 \\
\hline F6 & 0.50 & 1.60 & 0.25 & 68.74 & 17.5 & 21.8 & 76.4 \\
\hline F7 & 0.10 & 1.60 & 0.75 & 79.22 & 14.6 & 24.7 & 86.2 \\
\hline F8 & 0.50 & 1.60 & 0.75 & 90.13 & 16.1 & 27.8 & 79.3 \\
\hline F9 & 0.30 & 1.55 & 0.25 & 60.64 & 16.4 & 21.5 & 85.8 \\
\hline F10 & 0.30 & 1.65 & 0.25 & 56.54 & 24.2 & 23.7 & 90.4 \\
\hline F11 & 0.30 & 1.55 & 0.75 & 71.89 & 12.4 & 23.2 & 87.3 \\
\hline $\mathrm{F} 12$ & 0.30 & 1.65 & 0.75 & 63.33 & 22.0 & 23.1 & 85.7 \\
\hline $\mathrm{F} 13$ & 0.30 & 1.60 & 0.50 & 64.12 & 25.9 & 22.6 & 88.3 \\
\hline F14 & 0.30 & 1.60 & 0.50 & 62.89 & 25.8 & 22.5 & 88.6 \\
\hline $\mathrm{F} 15$ & 0.30 & 1.60 & 0.50 & 63.75 & 25.6 & 22.4 & 88.1 \\
\hline \multicolumn{5}{|c|}{ Variables } & Low & Medium & High \\
\hline \multicolumn{5}{|c|}{$\mathrm{X}_{1}=$ Capmul MCM (oil) } & $0.1 \mathrm{~mL}$ & $0.3 \mathrm{~mL}$ & $0.5 \mathrm{~mL}$ \\
\hline \multicolumn{5}{|c|}{$\mathrm{X}_{2}=$ Accenon CC: Transcutol P $\left(\mathrm{S}_{\mathrm{mix}}\right)$} & 1.55 & 1.60 & 1.65 \\
\hline \multicolumn{5}{|c|}{$\mathrm{X}_{3}=\%$ aqueous polycarbophil } & $0.25 \%$ & $0.5 \%$ & $0.75 \%$ \\
\hline
\end{tabular}

Amount of curcumin: $30 \mathrm{mg}$; total volume of $\mathrm{MMEC}=10 \mathrm{~mL}$

RT: Retention time (time required for formulations to separate from the agar plates), MMEC: Mucoadhesive microemulsion in curcumin, $\mathrm{S}_{\text {mix }}$ : Mixture of surfactant and co-surfactant 


\section{Evaluation of dependent variables}

Droplet size, zeta potential, and polydispersity index (PDI)

The droplet size, zeta potential, and PDI of optimized MMEC were determined by Zetasizer (nano Z.S.; Malvern Instruments Inc, Malvern, U.K.). Therefore, to identify the type of ME formed, a dilutability test was carried out by dispersing $1 \mathrm{~g}$ formulation in $100 \mathrm{~mL}$ of distilled water and was evaluated for phase separation. ${ }^{22}$

\section{The mucoadhesive potential}

The mucoadhesive potential, indicated by the residence time of developed nasal formulations, was evaluated as per reported method. ${ }^{23-25}$ Briefly, $100 \mathrm{mg} \mathrm{MMEC} \mathrm{was} \mathrm{kept} \mathrm{on} \mathrm{the}$ focal point of the different agar plates at room temperature at (1\% w/w, prepared in phosphate buffer solution, $\mathrm{pH} 6.4$ ). After $10 \mathrm{~min}$, the agar plates were linked with the USP disintegration test equipment and moved up and down at a speed of $30 \pm 2$ times in phosphate-buffered saline at $37 \pm 1^{\circ} \mathrm{C}$. The time taken by the formulations to isolate from the agar plates was noted outwardly as the residence time of the formulations. ${ }^{22}$

\section{Flux}

Flux was quantified as the amount of permeated curcumin from the unit area of the nasal mucosa. For this study, a Franz diffusion cell with an effective diffusion area of $7.06 \mathrm{~cm}^{2}$ and volume $30 \mathrm{~mL}$ was used. ${ }^{26-29}$ The sheep nasal mucosa with the same thickness was collected from slater house in formalin, prepared, and mounted on the receptor compartment with $30 \mathrm{~mL}$ of phosphate buffer $(\mathrm{pH}:$ 6.4) as diffusion medium. The donor compartment was amassed to it and was stacked with $1 \mathrm{~mL}$ MMECs, PCG, and plain curcumin solution (PCS) ( $\approx 3 \mathrm{mg}$ curcumin). Diffusion was done at $37 \pm 1^{\circ} \mathrm{C}$ and 50 rpm. At predetermined intervals of 10 mins, an aliquot of 0.5 $\mathrm{mL}$ diffusion medium was drawn from the receptor medium and was dissected by UV-VIS spectrophotometer at $422 \mathrm{~nm}$. The results are obtained three times and the mean value is considered.

\section{In vitro drug diffusion study}

In vitro drug diffusion study of MMEC was conducted in a modified dissolution apparatus containing $400 \mathrm{~mL}$ of dissolution media, i.e., phosphate buffer saline (PBS), $\mathrm{pH} 6.4 .^{30-35}$ The temperature was maintained at $37 \pm 1^{\circ} \mathrm{C}$ and set rpm at 50 . The dialysis membrane of cut-off weight $10.000 \mathrm{D}$ was soaked in PBS ( $\mathrm{pH}$ : 6.4) overnight before the experiment. Different formulations of MMEC, PCG, and PCS equivalent to $30 \mathrm{mg}$ curcumin were placed in separate diffusion bags tied to both ends. Aliquots ( $5 \mathrm{~mL}$ ) were withdrawn at an interval of $30 \mathrm{~min}$ for the first one hour and then $1 \mathrm{~h}$ intervals for the rest of the study period, i.e., $10 \mathrm{~h}$, and supplanted with a similar measure of new phosphate-buffered saline. After proper dilution with dilution media, samples were evaluated at $422 \mathrm{~nm}$ by UV-VIS spectrophotometer. ${ }^{30-34}$ The results were obtained thrice and the mean value was considered.
pH

$\mathrm{pH}$ of the optimal MMEC was measured using a digital $\mathrm{pH}$ meter (Welltonix digital $\mathrm{pH}$ meter PM100). One g of MMEC was accurately weighed and dispersed in $10 \mathrm{~mL}$ of purified water. The calibrated pHmeter electrode was inserted into the sample 10 min before reading at room temperature. $\mathrm{pH}$ value was measured in triplicate and the mean value was calculated. ${ }^{32}$

\section{The content analysis of drug}

For drug content, $0.5 \mathrm{~g}$ equivalent weight of curcumin was placed in a $100 \mathrm{~mL}$ volumetric flask and dissolved in $50 \mathrm{~mL}$ of ethanolic phosphate buffer $(70 \mathrm{~mL}$ ethanol and $30 \mathrm{~mL}$ PBS- $\mathrm{pH}$ : 6.4). The volumetric flask was maintained for $2 \mathrm{~h}$ and shaken well in a shaker to mix it properly. It was diluted appropriately and analyzed on a UV-VIS spectrophotometer (Shimadzu UV 1800) at $422 \mathrm{~nm}$.

\section{Spreadability}

For MMEC gel spreadability study, $0.5 \mathrm{~g}$ MMEC was placed on the glass plate within a premarked circle of $10 \mathrm{~mm}$ diameter. A second plate was put over this first petridish, and $50 \mathrm{~g}$ weight was permitted to rest on the upper petridish. ${ }^{2,26}$ Spreading of the gel with respect to the increase in the diameter was noted.

\section{Viscosity}

Viscosity of the developed MMEC was measured in triplicate at $25 \pm 1^{\circ} \mathrm{C}$ by brookfield viscometer (model HVT). ${ }^{2,32}$ The prepared MMEC $50 \mathrm{~g}$ was placed in a container and permitted to equilibrate for 5 min prior to estimation dial measurements using TC shaft spindle \#64 at 20 rpm.

\section{Transmission electron microscopy (TEM)}

Morphology of the emulsion droplet for the optimal MMEC was observed using TEM (JEM 1010, JEOL Ltd., Tokyo, Japan) with an acceleration voltage of $80 \mathrm{Kv}$. The optimal MMEC was diluted with water (1:1000). One drop of the sample was directly plain matrix with has a cross-section size of 300 , discolored to $2 \%$ weight by volume tungstic acid for $2 \mathrm{~min}$, after drying the MMEC. It was extra dried at retention time (RT) and then inspected using TEM. The interaction was determined at the image was intensified 1, 50.000 times at 8.000 times. $^{2,32}$

\section{Nasal ciliotoxicity}

The nasal ciliary toxicity study was conducted using extracted sheep nasal mucosa to evaluate the ingredients' safety to the mucosal layer used in the formulation. In a briefly, the nasal mucosa of the sheep except the diaphragm was collected from the slaughterhouse in phosphate buffered saline $(\mathrm{pH}: 6.4)$. At that point, three different parts of the nasal mucosa (NP1, NP2, NP3) having an equal thickness were mounted on a Franz dispersion cell and then were exposed to $2 \mathrm{~mL}$ MMEC (test sample, $2860 \mu \mathrm{g} / \mathrm{mL}$ curcumin), PBS ( $\mathrm{pH}$ 6.4) (negative control) and isopropyl alcohol, a serious nasal mucociliary toxicity agent (positive control) 23,26 for $2 \mathrm{~h}$, respectively. All three nasal samples were washed with purified water after $2 \mathrm{~h}$ and the cross-section of the mucociliary was examined with an optimal microscope (Nikon Fx35A, Japan) then the sample was stained with hematoxylin and eosin. $23,26,30$ 


\section{Animal experiment}

The rats were maintained at temperature $\left(25 \pm 2^{\circ} \mathrm{C}\right)$ and humidity ( $45 \pm 5 \%$ ) and were supplied with standard laboratory diet and water ad libitum on a $12 \mathrm{~h}$ light/dark cycle. The rats were assigned to five groups with six animals in each group.

Group I: Saline treated (normal control).

Group II: Intranasal administration of optimized MMEC at 2860 $\mu \mathrm{g} / \mathrm{kg}$ of body weight at two-hour intervals was administered through intraperitoneal route for two weeks.

Group III: Animals treated animal PCG $2860 \mu \mathrm{g} / \mathrm{kg}$ body weight at two-hour intervals were administered through intraperitoneal route for two weeks.

Group IV: Animals were first treated with MMEC $2860 \mu \mathrm{g} / \mathrm{kg}$ in the same above method followed by intranasal applied plain drug solution at $2860 \mu \mathrm{g} / \mathrm{kg}$ of curcumin/ $\mathrm{kg}$ of body weight for two weeks.

Group V: Animals were treated with $2860 \mu \mathrm{g} / \mathrm{kg}$ of curcumin $/ \mathrm{kg}$ of body weight for two weeks.

In brief, the animals were anesthetized using intraperitoneal pentobarbital injection ( $40 \mathrm{mg} / \mathrm{kg}$ of body weight) and were maintained their body temperature by keeping them on a heating pad set at $37^{\circ} \mathrm{C} .40 \mu \mathrm{L}$ MMEC and PCG containing curcumin equivalent to $2.86 \mathrm{mg} / \mathrm{kg}$ each were administered through the intranasal route with the assistance of a micropipette (200 $\mu \mathrm{L}$ ) connected to low-density polyethylene tubing with 0.1 $\mathrm{mm}$ inner measurement at the delivery site. For intravenous administration, $0.5 \mathrm{~mL}$ PCS was delivered (portion comparable to $2.86 \mathrm{mg} / \mathrm{kg}$ ) through the femoral vein. At 5, 15, 30, 60, 120, 180,240 , and 360 min after the portion, blood was gathered in the anesthetized condition by cardiac puncture in heparinized eppendorf tube (Eppendorf India Pvt. Ltd., Bangalore). Blood samples were then centrifuged at $6000 \mathrm{rpm}$ and $-4^{\circ} \mathrm{C}$ for 15 min to obtain plasma. At each time point, the animals have sacrificed by euthanasia. At that point, the skull was cut open, and the olfactory bulb (OB), olfactory plot (OT), CRB, and cerebellum (CEB) were carefully excised. All four excised brain tissues were rinsed with saline followed by blotting up with channel paper to take out blood spoil and plainly visible veins as much as could reasonably be expected. After gauging, the CRB tissue was homogenized independently with $5 \mathrm{~mL}$ saline in a tissue homogenizer (BD-144 tissue homogenizer, India). All tissue samples, such as aliquots of plasma and CRB tissue homogenates, were put away in a freezer $\left(-20^{\circ} \mathrm{C}\right)$ until HPLC analysis. Measurements of curcumin were made using 3 rats at each time point.

\section{Pharmacokinetic data analysis}

A non-compartmental pharmacokinetic analysis method was used to investigate the pharmacokinetic behavior of curcumin. Microsoft Excel was used to calculate the pharmacokinetic parameters from the experiments. The total area under the plasma concentration-time curve was determined by the trapezoidal rule using plasma curcumin concentration vs. time data from time zero to the last sampling time, i.e. $6 \mathrm{~h}$ plus the extrapolated area (from the last experimental time to infinity). The relative bioavailability of the representative MMEC to control was calculated as follows:

Relative bioavailability \%= $\left[\left(\mathrm{AUC}_{\mathrm{MMEC}} \times\right.\right.$ Dose $\left._{\text {control }}\right) /\left(\right.$ Dose $_{\mathrm{MMEC}} \times$ $\mathrm{AUC}_{\text {control }}$ ]

where;

Area under curve ${ }_{M M E C}\left(A \cup C_{M M E C}\right)$ means the area under the plot of plasma concentration of a drug versus time after MMEC gives insight into the extent of exposure to a MMEC and its clearance rate from the body.

The AUC control represents the total exposure to the curcumin solution across time.

The clear elimination half-life $\left(t_{1 / 2}\right)$ was calculated from the estimated elimination rate constant (kel) by linear regression of the log of the plasma concentrations as in $0.693 / \mathrm{kel}$. The elimination rate constant (kel) can be calculated directly from those parameters using the equation kel equals clearance divided by the volume of distribution. The maximum plasma concentration and time to maximum concentration after oral administration were determined directly from the concentration versus time curve.

\section{Analytical method}

Curcumin in plasma and brain tissue was quantified by HPLC method. ${ }^{27}$ Curcumin was extracted from plasma by protein precipitation method. ${ }^{28,36}$ To $0.2 \mathrm{~mL}$ of processed plasma samples, $10 \mu \mathrm{L}$ emodin (suitably diluting in methanol) was added as an internal standard and was sonicated in a bath sonicator for foe 120 seconds. The HPLC system consisted of an LC-10AD VP HPLC pump (Shimadzu, Japan) equipped with a UV detector with lab-solution software. The column used was an agilent C18 column (Inertsil, $250 \mathrm{~mm} \times 4.6 \mathrm{~mm}$, particle size $5 \mu$, USA). Chromatographic analysis were carried out at $1 \mathrm{~mL} / \mathrm{min}$ flow rate of the mobile phase i.e., acetonitrile - 5\% acetic acid (75:25, v/v). The mobile phase was prepared by mixing acetonitrile and $5 \%$ acetic acid and was further filtered by a $0.22 \mu$ membrane filter, followed by degassing by sonication before use. Elution of the drug was identified at 425 nanometers. $80-400 \mu \mathrm{L}$ of brain tissue homogenates, $30 \mu \mathrm{g} / \mathrm{mL}$ of emodin was added and stirred for $2 \mathrm{~min}$. At this point of centrifugation at $10.000 \mathrm{rpm}$ for 10 min, the drug was removed from the supernatant by adding $0.7 \mathrm{~mL}$ of $n$-hexane and diethyl ether (1:1). Next, the separated organic phase was evaporated at $40^{\circ} \mathrm{C}$ until dryness. The residue reconstituted in $100 \mu \mathrm{L}$ mobile phase, and afterward, $20 \mu \mathrm{L}$ was injected onto the HPLC framework. The residue was reconstituted in $100 \mu \mathrm{L}$ mobile phase, and then $20 \mu \mathrm{L}$ was implemented into an HPLC framework. ${ }^{2}$ The blood samples were analyzed using the same mobile phase and chromatographic conditions.

The RT was 5.8 min for curcumin and 3.2 min for the interior standard (i.e. emodin). The linear range of curcumin is 40$600 \mathrm{ng} / \mathrm{mL}$, and the linear range of plasma and brain tissue is $20-400 \mathrm{ng} / \mathrm{g}$. Extraction recoveries of curcumin from plasma and tissue homogenates were more than $86.7 \%$ and $82.6 \%$, respectively. ${ }^{33}$ 


\section{Stability study}

The stability of MMEC was carried out as per the International Conference on Harmonization guidelines (Q1A, R2) for 6 months ${ }^{30,36}$ the optimal ME was stored at cold temperature (4$\left.8^{\circ} \mathrm{C}, 45 \% \mathrm{RH} \pm 5\right),\left(25 \pm 2^{\circ} \mathrm{C}\right.$, and $\left.60 \% \mathrm{RH} \pm 5\right)$ and at accelerated temperature $\left(40 \pm 2{ }^{\circ} \mathrm{C}, 75 \% \mathrm{RH} \pm 5\right)$. After each 3 months for half year, MMEC was analyzed for droplet size, particle size distribution, mucoadhesion, and percent drug content. Since curcumin is reported to light-sensitive, the formulation was kept in an amber-colored container. ${ }^{37}$

\section{Statistical analysis}

All data were displayed as mean \pm standard deviation (SD), and the distinctions between the groups were tested using the student's $t$-test at the significance level of $p<0.05$. More than two groups were compared using ANOVA and differences greater at $p<0.05$ were considered significant.

\section{RESULTS AND DISCUSSION}

\section{Pre-formulation study}

Capmul MCM is a compatible hydrophobic vehicle to the nasal mucosa, which exhibiting maximum curcumin-dissolving volume $(42.17 \pm 3.12 \mathrm{mg} / \mathrm{mL})$ compared to other screened oils to develop a ME system. Therefore, it was designated as the oil phase. However, accenon CC, which has an HLB value of 15.6, was chosen as the surfactant and has relatively low drug solubility compared to other surfactants. The selection of capmul was MCM was based on more reservoirs and a more negligible effect partitioning effect of curcumin. Because curcumin remains in the core phase as a dissolved state, curcumin is less soluble in accenon CC, which leads to sustained drug release., ${ }^{7,30}$ The longer retention time of curcumin may be due to the loading of curcumin in MME as a reserve source for sustained release of curcumin in brain tissue. The results from the release studies confirm this controlled release. The accumulation of curcumin in the brain may be due to the microsizes of the particles and the presence of surfactant on MME surfaces, which may cause the brain uptake reduction of lipid carriers by the reticuloendothelial system. The predicted result can be compared to data obtained from the literature..$^{20}$ In his results, Bashara specified the controlled release of buspirone and high concentration of the same buspirone in the brain, which approving the transport of blunt nasal passages to the brain after an intranasal loading ME of buspirone. ${ }^{30}$

Results of the pseudo-ternary phase study showed that accenon $\mathrm{CC}$ and transcutol $\mathrm{P}\left(\mathrm{S}_{\text {mix }}\right)$ revealed no significant changes in the existing ME region from 2:1, 2.5:1, and 3:1. For developing ME, $S_{\text {mix }}$ of 2.5:1 was chosen since another two proportions (2:1 and $3: 1$ ) have more surfactant, which may not help the supply the reservoir properties of the formulation. Then, at that point, we obtained an appropriate ME, which did not aggravate the nasal mucosa, $\mathrm{S}_{\text {mix }}$ having relatively less surfactant was not selected. Pseudo-ternary phase diagram is shown in Figure 1. These data can be compared to the findings of the results in the literature. ${ }^{30-32}$

\section{Optimization of MMEC}

To develop the best MMEC and to observe the effect of independent variables on the responses, such as normal droplet size, flow rate, and retention time as mucosal adhesion possible and drug release (\%), three independent variables ( $X 1$, $\mathrm{X} 2$, and $\mathrm{X} 3$ ) is based on response surface method to Design Expert $^{\circledR}$ programming $^{14}$ and the consequences of various regression analysis are summed up in Table 2. Additionally, as shown in Table 1, we also see that the normal droplet size, transition, retention time, and curcumin drug discharge (\%) are significantly affected by the independent factors described in polynomial equations 1 to 4 .

The evaluation of the responses showed the suitability of the quadratic model ( $p$ <0.05). Final conditions for all responses, i.e., mean droplet size, movement, hold time, and drug discharge (\%) in terms of their respective coded values, were obtained by running ANOVA as follows.

Droplet size $=+77.88+3.47 \times A-2.63 \times B+3.89 \times C-0.64 \times(A$ $\times B)+1.12 \times(B \times C)+2.54 \times(A \times C)+1.52 \times A^{2}-1.67 \times B^{2}+2.37$ $\times \mathrm{C}^{2}$

equation (1)

Equation 1 showed that the average droplet size of MMEC was generally influenced by capmul MCM, accenon CC: transcutol $\mathrm{P}$ concentration ratio and polycarbophil with their interactions. For developing a suitable intranasal drug delivery system, droplet size plays a vital role as it affects the in vivo absorption of the drug from the formulations. ${ }^{4,24}$ The droplet size of the ME formulation is a key factor because it affects the release rate of the drug and the in vivo profile of the drug. In this way, the experiment of optimizing a ME with a droplet size usually smaller confirms that the rapid penetration through mucous layers is an objective of this research. The observed droplet size of the formulations ranged between $55.82 \mathrm{~nm}$ and $90.13 \mathrm{~nm}$ (Table 1). The results showed that the increase in the oil volume from 0.1 $\mathrm{mL}$ to $0.5 \mathrm{~mL}$ and the concentration of the mucosal adhesion polymer from $0.25-0.75 \%$ caused a significant increase in globule size of ME formulation. This could be because it did not diminish the interfacial tension among the oil and the external phase with maximum concentration with a similar mix. Additional, at higher $\mathrm{S}_{\text {mix }}$ concentration, the lipophilic property of oil was well masked, resulting in low interfacial tension, and therefore, droplet size reduces. ${ }^{23}$ Mucoadhesive polymer also shows to upsurge the droplet size, which may be because it was capable of absorbing water and swell, which in turn disturbed the HLB of the system.

Flux $=+88.46+2.47 \times B-1.83 \times C+0.58 \times(A \times B)-1.67 \times(A \times$ C) $+1.55 \times C^{2}$ equation (2)

Capmul MCM due to its reservoir action and polycarbophil due to the viscosity enhancing property showed a negative effect on the release rate. Therefore, the drug concentration gradient across the permeation barrier (nasal mucosa) is not high and hence the flux. The results obtained in this study indicated that oil concentration (X1) and mucoadhesive polymer concentration (X3) have significant effects on the flux through sheep nasal 
mucosa $(p-0.0001)$. However, $\mathrm{S}_{\text {mix }}$ showed a positive effect on flux because both surfactant and cosurfactant are capable of altering the permeation behavior of the membrane by changing the fluidization of lipid enabling the drug molecule to permeate through rapidly.

Retention time $=+47.37+3.55 \times C+2.79 \times(A \times C)+0.88 \times(B \times$ C) $-0.76 \times B^{2}+1.04 \times C^{2}$ equation (3)

Polycarbophil, due to its mucoadhesive property along with viscosity enhancing property, showed a noticeably positive effect on the retention time of MMEC on the nasal mucosa, while oil and $\mathrm{S}_{\text {mix }}$ showed non-significant effects as shown in equation 3 . The mucoadhesive nature of the polymer may be because of the presence of a high density of hydrogen bonding groups, which could combine with mucin more strongly, as shown in equation $3(p-0.0021) .^{37}$

Drug release $(\%)=+93.87-1.89 \times A+2.01 \times B-3.11 \times C+0.88 \times$ $(A \times B)-1.95 \times(A \times C)-1.89 \times(B \times C)+0.51 \times A^{2}-0.67 \times B^{2}-1.34$ $\times C^{2}$

equation (4)

Drug release from MMEC was inversely affected by capmul MCM and polycarbophil concentration, while a mixture of accenon CC and transcutol $P$ was found to facilitate the release of curcumin from the developed formulation, as revealed in equation 4. Drug release from the model MMEC batches after $8 \mathrm{~h}$ ranged from $79 \%$ to $96 \%$, as given in Table 1. Capmul MCM showed to retard

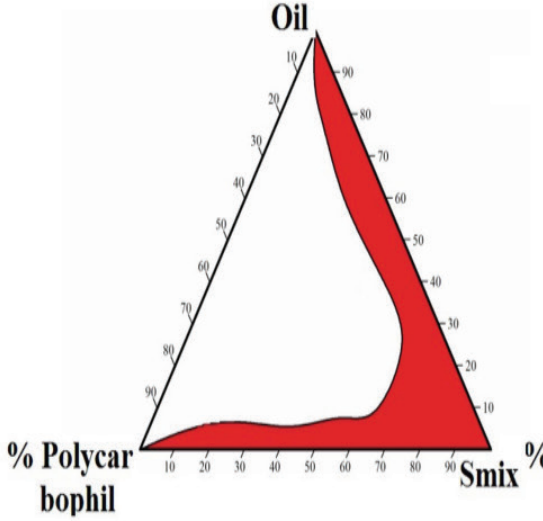

Smix- 2:1

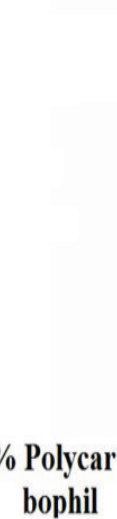

bophil

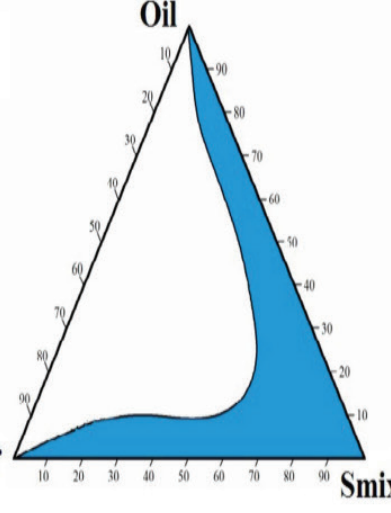

Smix $-2.5: 1$

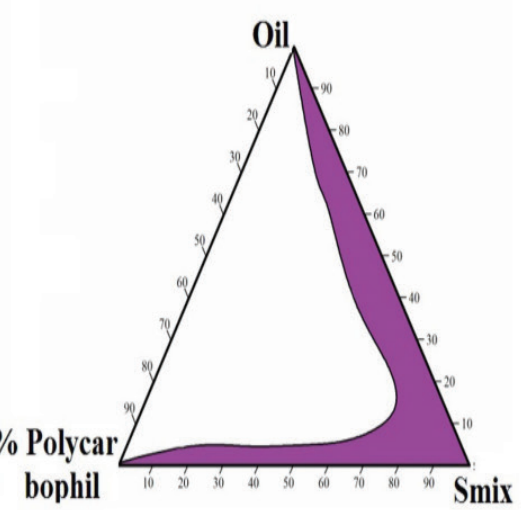

Smix- 3:1

Figure 1. Pseudo-ternary phase diagram of MMEC showing microemulsion existing region with 2.5:1 $\mathrm{S}_{\text {mix }}$ MMEC: Mucoadhesive microemulsion in curcumin

Table 2. Statistical parameter of responses determined by multiple regression analysis

\begin{tabular}{|c|c|c|c|c|}
\hline \multirow{2}{*}{ Regression coefficient } & \multicolumn{4}{|c|}{ Coefficient estimate } \\
\hline & Globule size $(\mathrm{nm})$ & Flux $\left(\mu \mathrm{g} / \mathrm{cm}^{2} \mathrm{~h}\right)$ & $\mathrm{Rt}(\min )$ & Drug release (\%) \\
\hline A- Capmul MCM (X1) & 3.47 & -0.47 & 0.39 & -1.89 \\
\hline$B-S_{\text {mix }}(X 2)$ & -2.63 & 2.47 & -0.21 & 2.01 \\
\hline C- \% aqueous polycarbophil (X3) & 3.89 & -1.83 & 3.55 & -3.11 \\
\hline$A B(X 1 X 2)$ & -0.64 & 0.58 & 0.35 & 0.88 \\
\hline$A C(X 1 X 2)$ & 2.54 & -1.67 & 2.79 & -1.95 \\
\hline$B C(X 1 X 2)$ & 1.12 & -0.43 & 0.88 & -1.89 \\
\hline$A^{2}$ & 1.52 & -0.45 & 0.37 & 0.51 \\
\hline$B^{2}$ & -1.67 & 0.43 & -0.76 & -0.67 \\
\hline $\mathrm{C}^{2}$ & 2.37 & 1.55 & 1.04 & -1.34 \\
\hline Model ( $p$ value) & 0.0002 & 0.0003 & 0.0021 & 0.0002 \\
\hline Coefficient of variation & 0.991 & 0.999 & 0.962 & 0.999 \\
\hline $\mathrm{R}^{2}$ & 0.973 & 0.998 & 0.976 & 0.997 \\
\hline Adjusted $\mathrm{R}^{2}$ & 0.6710 & 0.2891 & 0.8771 & 0.0580 \\
\hline Lack of fit ( $p$ value) & 3.47 & -0.47 & 0.39 & -1.89 \\
\hline
\end{tabular}

Rt: Retention time, $\mathrm{S}_{\text {mix }}$ : Mixture of surfactant and co-surfactant 
the drug release from the formulation due to its partitioning and reservoir properties, while polycarbophil because of its viscosity enhancing property, kinetics of the drug molecule reduced leading to the slow release. However, $\mathrm{S}_{\text {mix }}$ (accenon CC and transcutol $P$ mixture) increased curcumin release because it increased the water solubility of curcumin by reducing the interfacial tension. This increase in drug release may be further be due to nanoglobule size with a narrow size distribution.

The rationale of optimization through factorial design was to obtain the ranges of all independent factors, establish their influence on responses, and find a robust composition for intranasal delivery of curcumin. In this study, globule size, viscosity, and retention time (Rt indicating the mucoadhesive property) were set to the maximum without affecting release, while flux and release were set to maximum. Confirming the desirability of the optimized nasal formulations, three MMECs, so obtained from the overlay plot were prepared experimentally, and all responses were evaluated as given in Table 3. It was observed that experimentally found data matched the predicted responses for all three MMECs, and hence, the optimization process was verified. ${ }^{32-34}$

\section{MMEC characterization}

MMEC with $0.5 \% \mathrm{w} / \mathrm{v}$ polycarbophil, $0.3 \mathrm{~mL}$ capmul MCM, $3.70 \mathrm{~mL} \mathrm{~S}_{\text {mix }}$ (2.5: 1 ratio) exhibited the smallest droplet size (55.82-90.12 nm); the highest flux and drug release (80-96.1\%) are demonstrated in Table 3 and are closer to the observed results than predicted. Therefore, was considered the optimal formulation. The average globule size was $66.74 \pm 3.46 \mathrm{~nm}$ with PDI equivalents to $0.133 \pm 0.017$, as revealed in Figure 2. Besides, the PDI value $(<0.3)$ showed the monodisperse property of the formulation. ${ }^{38}$

Zeta potential of MMEC was $-21.4 \mathrm{mV} \pm 4.11$ as shown in Figure 3. These data depict neither the stability of the formulation, which might be because moderate negative surface charges neither result in strong aggregation nor repulsion of the globules. ${ }^{7}$ So, individual nanoglobules increased the surface area, which in turn helped in the nasal absorption of the drug and hence the stability of formulation and brain targeting. TEM data further support the nanosize with narrow size distribution, as illustrated in Figure 4.

Curcumin content was discovered to be $97.82 \pm 0.44 \%, 98.11 \pm$ $0.51 \%$ and $99.32 \pm 0.22 \%$ for MMEC, PCG and PCS individually. The $\mathrm{pH}(6.7 \pm 0.18)$, thickness, and mucoadhesive strength data showed the appropriateness for the nasal application of MMEC. The stability studies showed no significant changes in droplet size; size distribution, retention time, and phase separation over six months were observed from the stability study. Hence, developed mucoadhesive nasal formulation of curcumin was considered physically stable for 6 months.

Optical microscopic pictures showed no mucociliary damage by MMEC as shown in Figure 5C, which revealed non-ciliotoxicity profile of developed MMEC while complete cilia destruction was observed in the isopropyl-treated mucosa. Hence, developed MMEC was considered suitable for nasal application. This may be because all formulation compositions were of GRAS and had no interaction.

The prepared gel produces excellent spreadability and mucoadhesive strength. The drug content of the MMEC was found to be $98.67 \pm 0.44 \%$ and $\mathrm{pH}$ was found to be $6.58 \pm 0.29$. The viscosity of the gel was found to be $18.7 \pm 2.11 \mathrm{Ps}$ at $10 \mathrm{rpm}$. Spreadability, $\mathrm{pH}$, viscosity, and mucoadhesive strength data show the suitability for intranasal application of MMEC. From the stability study, as shown in Table 4, no significant changes in globule size, size distribution, Rt, and phase separation over a period of 6 months were observed. Hence, developed mucoadhesive nasal formulation of curcumin was considered physical stable for 6 months.

\section{Pharmacokinetic study}

Curcumin concentration in plasma and brain tissue concentrations after nasal administration of MMEC and PCG and intravenous administration of PCS at $2.86 \mathrm{mg} / \mathrm{kg}$ body weight of rat was estimated. The pharmacokinetic parameters

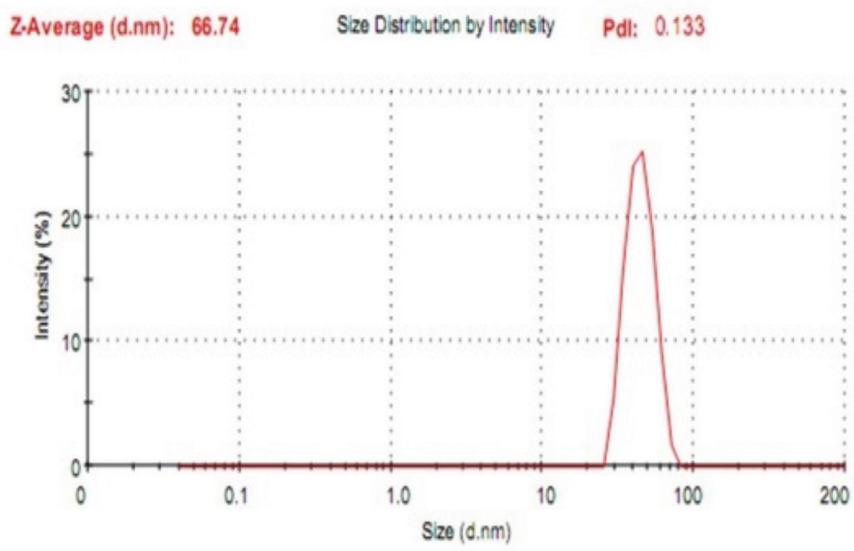

Figure 2. Result of globule size with size distribution indicating the nano size range of optimal MMEC

MMEC: Mucoadhesive microemulsion in curcumin

Table 3. Obtained predicted data and observed data of size, flux, retention time and drug release (\%) of MMEC

\begin{tabular}{|c|c|c|c|c|c|c|c|c|c|c|c|}
\hline \multirow{2}{*}{ No } & \multicolumn{3}{|c|}{ Components } & \multicolumn{2}{|c|}{ Globule size (nm) } & \multicolumn{2}{|c|}{ Flux $\left(\mu \mathrm{g} / \mathrm{cm}^{2} \mathrm{~h}\right)$} & \multicolumn{2}{|c|}{ Rt (min) } & \multicolumn{2}{|c|}{ Drug release $(\%)$} \\
\hline & 0 & $S_{\text {mix }}$ & $\mathrm{p}$ & Pre. & Obs. & Pre. & Obs. & Pre. & Obs. & Pre. & Obs. \\
\hline 1 & 0.30 & 3.80 & 0.50 & 48.6 & 47.4 & 24.0 & 23.9 & 22.9 & 22.2 & 93.9 & 95.6 \\
\hline 2 & 0.31 & 3.80 & 0.52 & 48.4 & 47.5 & 24.7 & 23.8 & 22.8 & 22.0 & 89.8 & 93.0 \\
\hline 3 & 0.30 & 3.70 & 0.50 & 46.7 & 46.3 & 25.4 & 25.8 & 22.9 & 22.8 & 94.1 & 94.8 \\
\hline
\end{tabular}

MMEC: Mucoadhesive microemulsion in curcumin, $\mathrm{S}_{\text {mix }}$ : Mixture of surfactant and co-surfactant, Rt: Retention time, O: Oil, Pre.: Predicted, Obs.: Observed 
obtained by non-compartmental pharmacokinetic analysis ( $\mathrm{n}$ : 3; mean \pm SD) are given in Table 5 .

Following the IV administration of PCS, plasma curcumin fixations arrived at the maximum level before $15 \mathrm{~min}$ and then decreased rapidly over time, as shown in Figure 6. After intranasal administration of MMEC, maximum concentration of curcumin was achieved even before $30 \mathrm{~min}$ in OB, while 60120 min in other brain parts like OT, CRB and CEB. Curcumin content differed considerably in different brain regions. The highest concentration was observed in the $\mathrm{OB}$ (the peak drug level was $1109.1 \pm 56.4 \mathrm{ng} / \mathrm{g}$ ), followed by OT, then the CEB and finally in CRB as shown in Figure 6. These findings support

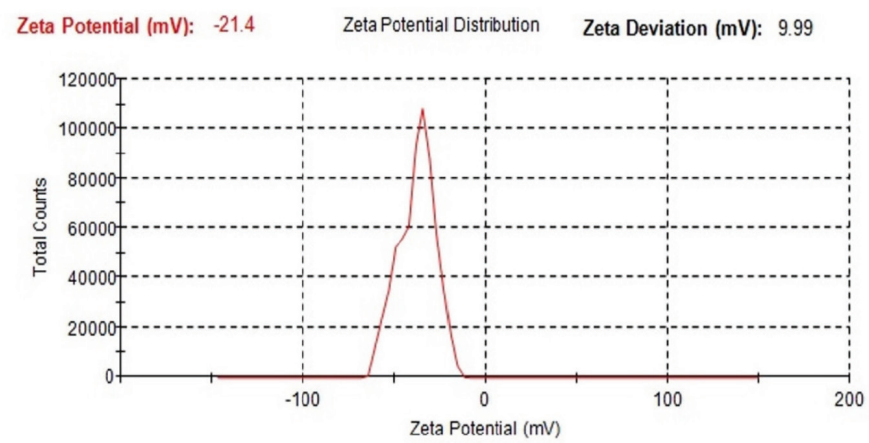

Figure 3. Zetapotential data representing the physical stability of MMEC MMEC: Mucoadhesive microemulsion in curcumin the existence of a nose-brain direct pathway following the intranasal administration. As shown in Table 5, curcumin concentration, particularly in the $\mathrm{OB}$, was noticeably higher than in other tested parts of the brain following nasal administration of MMEC.

AUCO $\rightarrow 360$ for MMEC was 2.81 times $(56449 \pm 3113 \mathrm{ng} \mathrm{min} / \mathrm{g})$ more that obtained after i.v. injection of PCS $(20088 \pm 1241 \mathrm{ng}$ $\mathrm{min} / \mathrm{g}$ ). However, PCG showed comparatively less curcumin uptake than that of MMEC, which was a clear indication of only mucoadhesive property was not sufficient for nasal delivery to the brain.

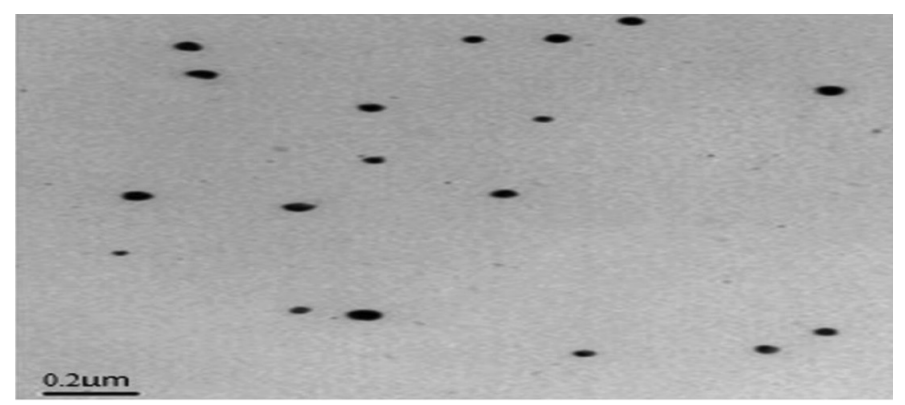

Figure 4. TEM result of the optimized MMEC indicating the narrow particle size with uniform distribution

TEM: Transmission electron microscopy, MMEC: Mucoadhesive microemulsion in curcumin

\section{Table 4. Results indicating the stability of developed nasal formulation in three different storage conditions}

\begin{tabular}{|c|c|c|c|c|c|c|c|c|}
\hline \multirow{3}{*}{$\begin{array}{l}\text { Temperature and } \\
\text { relative humidity }\end{array}$} & \multicolumn{8}{|c|}{ Evaluated parameters of MMEC } \\
\hline & \multicolumn{2}{|c|}{ Globule size $(\mathrm{nm})$} & \multicolumn{2}{|c|}{ Retention time (min) } & \multicolumn{2}{|l|}{ PDI } & \multicolumn{2}{|c|}{ Phase separation } \\
\hline & $3^{\text {rd }}$ month & $6^{\text {th }}$ month & $3^{\text {rd }}$ month & $6^{\text {th }}$ month & $3^{\text {rd }}$ month & $6^{\text {th }}$ month & $3^{\text {rd }}$ month & $6^{\text {th }}$ month \\
\hline $\begin{array}{l}2-8^{\circ} \mathrm{C} \\
45+5 \%\end{array}$ & $75.5 \pm 4.2$ & $83.3 \pm 3.7$ & $19.8 \pm 2.2$ & $22.3 \pm 1.9$ & $0.31 \pm 0.02$ & $0.36 \pm 0.07$ & No & No \\
\hline $\begin{array}{l}25+2^{\circ} \mathrm{C} \\
60+5 \%\end{array}$ & $62.7 \pm 4.8$ & $69.6 \pm 5.3$ & $22.5 \pm 1.27$ & $22.8 \pm 1.85$ & $0.201 \pm 0.02$ & $0.217 \pm 0.03$ & No & No \\
\hline $\begin{array}{l}45+2^{\circ} \mathrm{C} \\
75+5 \%\end{array}$ & $63.9 \pm 4.9$ & $61.8 \pm 4.4$ & $18.9 \pm 1.87$ & $20.1 \pm 1.37$ & $0.364 \pm 0.21$ & $0.343 \pm 0.017$ & No & Yes \\
\hline
\end{tabular}

(Result= mean \pm SD, n: 3). MMEC: Mucoadhesive microemulsion in curcumin, PDI: Polydispersity index, SD: Standard deviation

Table 5. Pharmacokinetic parameters of curcumin after transnasal and intravenous application of MMEC and PCS respectively ( $\mathrm{n}$ : 3 )

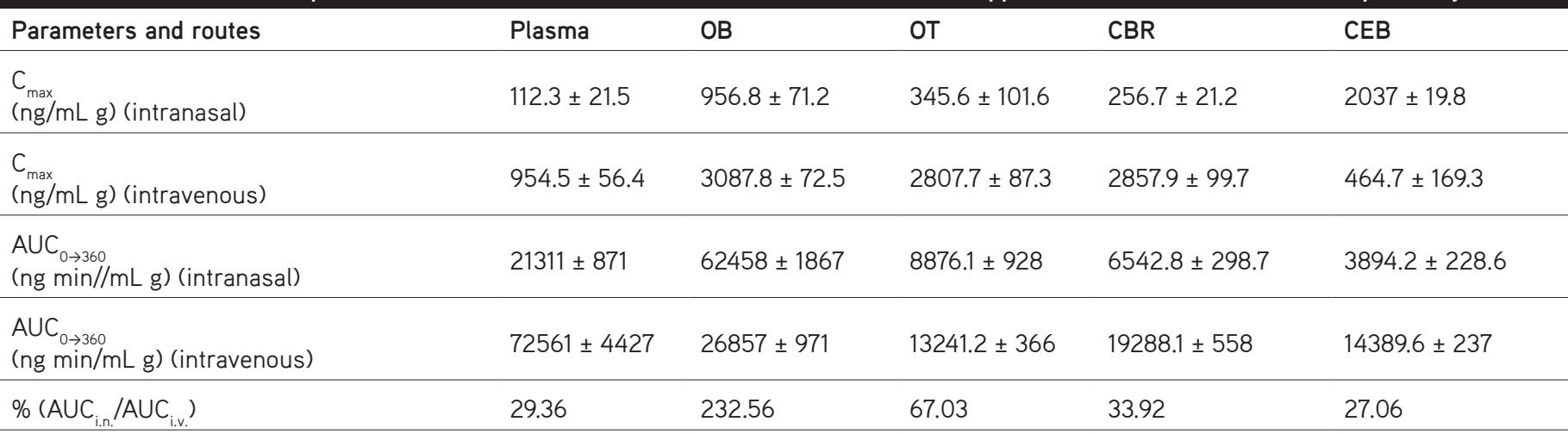

(Result; mean \pm SD, n: 3). $C_{\max }$ : Maximum peak plasma concentration, SD: Standard deviation, MMEC: Mucoadhesive microemulsion in curcumin, PCS: Plain curcumin solution, OB: Olfactory bulb, OT: Olfactory tract, CBR: Cerebrum, CEB: Cerebellum, AUC: Area under curve, i.v.: Intravenous, i.n.: Intranasal 


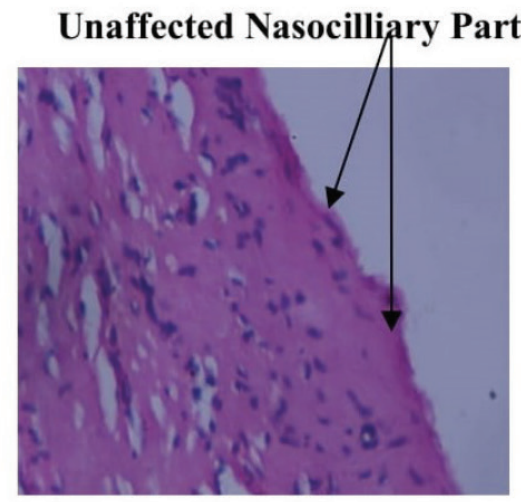

(5A)

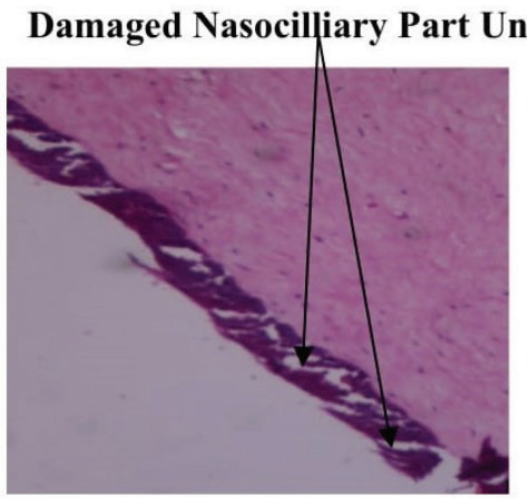

5(B)

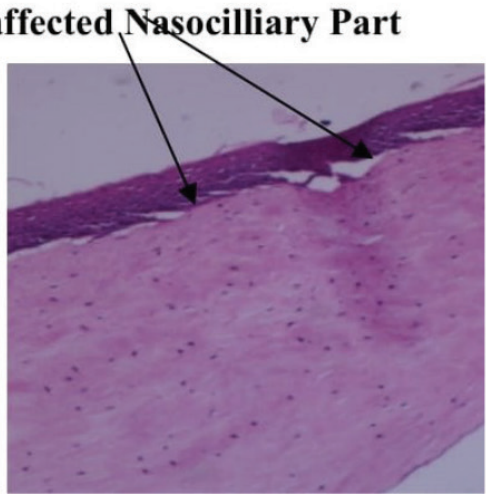

5(C)

Figure 5. Result of naso-ciliotoxicity study displaying the non-toxicity of developed MMEC. 5(A), 5(B), and 5(C) are representative of PBS ( $\mathrm{pH}$ : 6.4 ), propranolol and developed MMEC treated naso mucosal part individually

MMEC: Mucoadhesive microemulsion in curcumin, PBS: Phosphate buffer saline

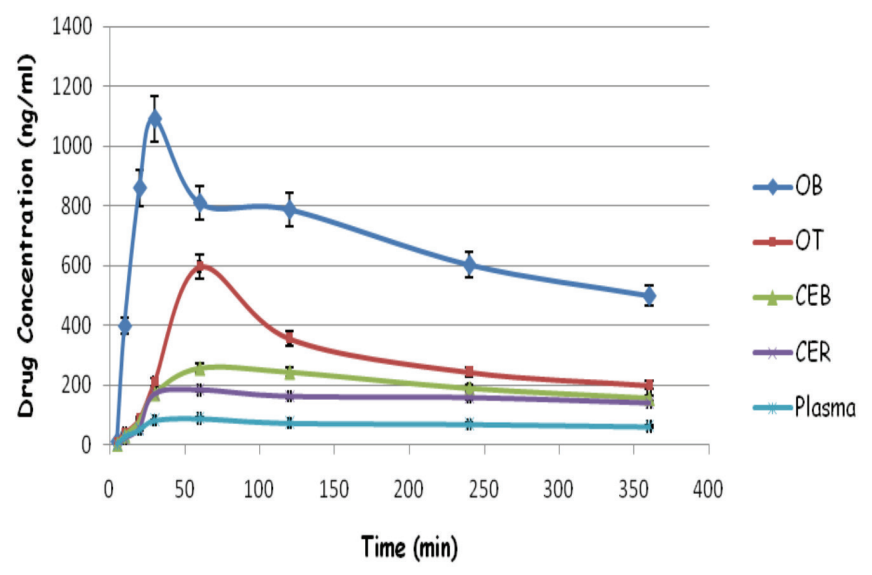

Figure 6. Curcumin concentration in different parts of brain and blood after intranasal delivery ( $n: 3$; mean \pm SD)

SD: Standard deviation, OB: Olfactory bulb, OT: Olfactory tract, CER: Cerebrum, CEB: Cerebellum

Curcumin uptake in other brain parts (OT, CRB, and CEB) after nasal dosing of MMEC was lower than $\mathrm{OB}$ ( $n$ : 3; mean \pm SD) as shown in Table 5 and Figure 6, which may be due to its anatomical position from the nasal turbinates. ${ }^{33,35}$ After nasal dosing of MMEC, curcumin transport from the nasal turbinates into the $\mathrm{OR}$ may occur through three pathways, i.e., transcellular between the sustentacular cells by endocytosis, paracellularly through tight junctions between the sustentacular cells, and intracellular axonal transport via the olfactory nerve pathway following endocytosis or pinocytosis into the $\mathrm{OB}$. MMEC increased the aqueous solubility of lipophilic curcumin, enhancing the drug permeation through the nasal cells by combining all three above described methods and reaching the therapeutic area. ${ }^{37,38}$

Curcumin uptake into other brain regions like OT, CBR, and CEB, after nasal dosing of MMECs was lower than i.v. dosing of PCS, as shown in Table 5 and Figure 6. These may be attributed to the comparative lower plasma levels from MMEC, which might reduce curcumin distribution in the brain. In contrast to CBR and CEB, the mass of the $O B$ is small. The distribution of higher curcumin from $\mathrm{OB}$ into other brain regions could be counteracted by its small masses, thus leading to a significant increase in curcumin content in different brain tissue (olfactory pathway). Due to the controlled release of curcumin from MMEC and mucoadhesive nature, prolonged curcumin absorption was observed, indicating the nose-direct brain transport. These obtained data were also similar to the data given in the literature ${ }^{36-38}$ showing a unique nasal mucosabrain connection. To evaluate the brain targeting through nasal delivery of MMEC, the brain-to-plasma curcumin AUC ratios at 10, 30, and 360 min following intravenous and intranasal routes were calculated. Results showed that the ratio of AUC in brain tissue to that in plasma after intranasal application of MMEC was significantly higher $(p<0.05)$ than those after intravenous injection of PCS and PCG. For instance, in $10 \mathrm{~min}$ after nasal dosing, the AUC ratio was 5.83 times higher than that after intravenous dosing (19.85 $\pm 1.5 \mathrm{v} / \mathrm{s} 3.4 \pm 1.1)$ in $\mathrm{OB}$, as shown in Figure 7A.

Similarly, 9.17 times $(60.41 \pm 2.8 \mathrm{v} / \mathrm{s} 6.58 \pm 1.3)$ and 9.56 times $(31.11 \pm 1.6 \mathrm{v} / \mathrm{s} 3.25 \pm 1.01)$ increment of curcumin concentration in $\mathrm{OB}$ following i.n. and i.v. delivery at the time points $30 \mathrm{~min}$ and 360 min ( $n: 3$; mean $\pm S D$ ) respectively as shown in Figure 7B, C. Following intranasal administration, $87.22 \%$ of curcumin was transported to the brain via the olfactory pathway in six hours. ${ }^{37,38}$ It can be concluded that the nasal route of administration and mucoadhesive formulation may help curcumin enhance its brain uptake.

\section{CONCLUSION}

Results confirmed that polycarbophil-based MME system including capmul MCM (3\% v/v), accenon CC $(26 \% \mathrm{v} / \mathrm{v})$, and transcutol $P(9 \% \mathrm{v} / \mathrm{v})$ was optimal for transnasal delivery of curcumin. The developed ME system was non-ciliotoxic, physical stable at ambient conditions for 6 months and was suitable for controlled curcumin delivery to the brain. Results of a brain distribution study confirmed that a fraction of 

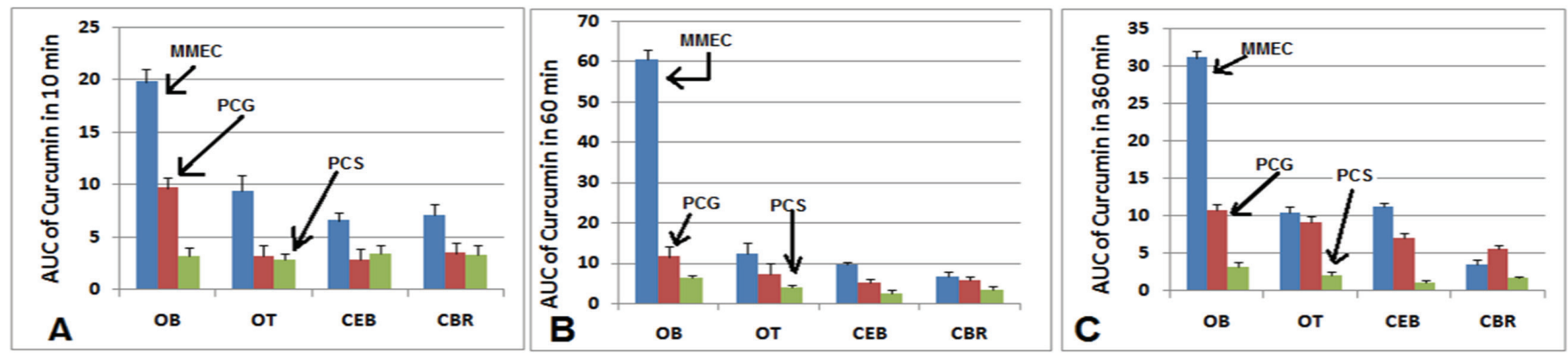

Figure 7. Plasma curcumin concentration and in different parts of brain after intranasal administration of MMEC, PCG and intravenous dosing of PCS after $10 \min (A), 30 \min (B)$, and 360 min (C) respectively ( $n: 3$; mean \pm SD)

MMEC: Mucoadhesive microemulsion in curcumin, PCG: Polycarbophil gel, PCS: Plain curcumin solution, SD: Standard deviation, OB: Olfactory bulb, OT: Olfactory tract, CBR: Cerebrum, CEB-Cerebrellum

curcumin could be transported directly into the brain after nasal delivery, which may decrease the dose and frequency of dosing and hence maximize its therapeutic index. However, clinical benefits to risk ratio of this mucoadhesive formulation so developed in this investigation will decide its effectiveness in the clinical practice.

\section{Ethics}

Ethics Committee Approval: The animal experiment was approved by the Institutional Animal Ethical Committee (CPCSEA No. 984/14/10/CPCSEA New Delhi, India) and registration no: 1244/08/ CPCSEA, India.

Informed Consent: Not necessary.

Peer-review: Externally peer-reviewed.

\section{Authorship Contributions}

Surgical and Medical Practices: J.P., Concept: S.D.M., J.P., S.M., Design: S.D.M., S.M., Data Collection or Processing: S.M., S.F., S.D.M., Analysis or Interpretation: S.F., Literature Search: M.S.P., S.D.M., S.M., S.F., J.P., Writing: M.S.P.

Conflict of Interest: No conflict of interest was declared by the authors.

Financial Disclosure: The authors declared that this study received no financial support.

\section{REFERENCES}

1. Romeo VD, deMeireles J, Sileno AP, Pimplaskar HK, Behl CR. Effects of physicochemical properties and other factors on systemic nasal drug delivery. Adv Drug Deliv Rev. 1998;29:89-116.

2. Mandal S, Mandal SD, Chutani K, Subudhia BB. The mucoadhesive microemulsion of ibuprofen: design and evaluation for brain targeting efficiency through intranasal route. Braz J Pharma Sci. 2015:51:721-731.

3. Mandal SD, Mandal S, Patel J. Brain targeting efficiency of curcumin loaded mucoadhesive microemulsion through intranasal route. J Pharm Investig. 2016;46:179-188.

4. Pathak R, Dash RP, Misra M, Nivsarkar M. Role of mucoadhesive polymers in enhancing delivery of nimodipine microemulsion to brain via intranasal route. Acta Pharm Sin B. 2014:4:151-160.

5. Aggarwal BB, Harikumar KB. Potential therapeutic effects of curcumin, the anti-inflammatory agent, against neurodegenerative, cardiovascular, pulmonary, metabolic, autoimmune and neoplastic diseases. Int J Biochem Cell Biol. 2009;41:40-59.

6. Lawrence MJ, Rees GD. Microemulsion-based media as novel drug delivery systems. Adv Drug Deliv Rev. 2000;45:89-121.

7. Tenjarla S. Microemulsions: an overview and pharmaceutical applications. Crit Rev Ther Drug Carrier Syst. 1999;16:461-521.

8. Mandal S, Mandal SD. Design and development of carbamazepine mucoadhesive microemulsion for intranasal delivery: an ex-vivo study. Int J Pharm Sci Rev Res. 2010;3:56-60.

9. Mandal SD, Mandal S, Patel J. Development of curcumin loaded microemulsion drug delivery system for improving its dissolution profile. IJPFA. 2013;4:101-107.

10. Himesh S, Sharan PS, Mishra K, Govind N, Ak S. Qualitative and quantitative profile of curcumin from ethanolic extract of Curcuma longa. Int Res J Pharm. 2011;2:180-184.

11. Bitter C, Suter-Zimmermann K, Surber C. Nasal drug delivery in humans. Curr Probl Dermatol. 2011;40:20-35.

12. Stevens J, Ploeger BA, van der Graaf PH, Danhof M, de Lange EC. Systemic and direct nose-to-brain transport pharmacokinetic model for remoxipride after intravenous and intranasal administration. Drug Metab Dispos. 2011;39:2275-2282.

13. Md S, Khan RA, Mustafa G, Chuttani K, Baboota S, Sahni JK, Ali J. Bromocriptine loaded chitosan nanoparticles intended for direct nose to brain delivery: pharmacodynamic, pharmacokinetic and scintigraphy study in mice model. Eur J Pharm Sci. 2013;48:393-405.

14. Paradkar A, Ambike AA, Jadhav BK, Mahadik KR. Characterization of curcumin-PVP solid dispersion obtained by spray drying. Int J Pharm. 2004;271:281-286.

15. Dhuria SV, Hanson LR, Frey WH 2nd. Intranasal delivery to the central nervous system: mechanisms and experimental considerations. J Pharm Sci. 2010;99:1654-1673.

16. Jeffrey $P$, Summerfield $S$. Assessment of the blood-brain barrier in CNS drug discovery. Neurobiol Dis. 2010;37:33-37.

17. Tsai YM, Chien CF, Lin LC, Tsai TH. Curcumin and its nano-formulation: the kinetics of tissue distribution and blood-brain barrier penetration. Int J Pharm. 2011;416:331-338.

18. Shah $B$, Khunt D, Bhatt $H$, Misra M, Padh $H$. Application of quality by design approach for intranasal delivery of rivastigmine loaded solid lipid nanoparticles: effect on formulation and characterization parameters. Eur J Pharm Sci. 2015;78:54-66. 
19. Swamy NGN, Abbasb Z. Mucoadhesive in situ gels as nasal drug delivery systems: an overview. Asian J Pharm Sci. 2012;7:168-180.

20. Wang X, Chi N, Tang X. Preparation of estradiol chitosan nanoparticles for improving nasal absorption and brain targeting. Eur J Pharm Biopharm. 2008;70:735-740.

21. Mandal SD, Mandal S, Patel J. Intranasal mucoadhesive microemulsion for the neuroprotective effect of curcumin in MPTP induced Parkinson model. Braz J Pharm Sci. 2017;53:e15223.

22. Aqil M, Kamran M, Ahad A, Imam SS. Development of clove oil-based nanoemulsion of olmesartan for transdermal delivery: Box-Behnken design optimization and pharmacokinetic evaluation. J Mol Liq. 2016;214:238-248.

23. Thakkar H, Patel A, Chauhan N. Formulation and optimization of mucoadhesive microemulsion containing mirtazapine for intranasal delivery. Chron Young Sci. 2014;4:25-32.

24. Joshi HM, Bhumkar DR, Joshi K, Pokharkar V, Sastry M. Gold nanoparticles as carriers for efficient transmucosal insulin delivery. Langmuir. 2006;22:300-305.

25. Kan P, Chen ZB, Kung RY, Lee CJ, Chu IM. Study on the formulation of o/w emulsion as carriers for lipophilic drugs. Colloids Surf B: Biointerfaces. 1999;15: 117-125.

26. Acharya SP, Pundarikakshudu K, Panchal A, Lalwani A. Preparation and evaluation of transnasal microemulsion of carbamazepine. Asian $J$ Pharm Sci. 2013;8:64-70.

27. Kumar M, Misra A, Babbar AK, Mishra AK, Mishra P, Pathak K. Intranasal nanoemulsion based brain targeting drug delivery system of risperidone. Int J Pharm. 2008;358:285-291.

28. Li J, Jiang Y, Wen J, Fan G, Wu Y, Zhang C. A rapid and simple HPLC method for the determination of curcumin in rat plasma: assay development, validation and application to a pharmacokinetic study of curcumin liposome. Biomed Chromatogr. 2009;23:1201-1207.

29. Wang D, Hu J, Lv L, Xia X, Liu J, Li X. Enhanced inhibitory effect of curcumin via reactive oxygen species generation in human nasopharyngeal carcinoma cells following purple-light irradiation. Oncol Lett. 2013;6:81-85.

30. Bshara HN, Ahmed RO, Holayel SM, El-Shamy AEH. Improvement of the bioavailability of buspirone $\mathrm{HCl}$ using intranasal delivery systems. $\mathrm{Az} \mathrm{J}$ Pharm Sci. 2012;45:86-102.

31. Karasulu HY, Sanal ZE, Sözer S, Güneri T, Ertan G. Permeation studies of indomethacin from different emulsions for nasal delivery and their possible anti-inflammatory effects. AAPS PharmSciTech. 2008;9:342348.

32. Kaur P, Kim K. Pharmacokinetics and brain uptake of diazepam after intravenous and intranasal administration in rats and rabbits. Int $\mathrm{J}$ Pharm. 2008;364:27-35.

33. Rao J, McClements DJ. Formation of flavor oil microemulsions, nanoemulsions and emulsions: influence of composition and preparation method. J Agric Food Chem. 2011;59:5026-5035.

34. Dey S, Mahanti B, Mazumder B, Malgope A, Dasgupta S. Nasal drug delivery: an approach of drug delivery through nasal route. Der Pharmacia Sinica. 2011;2:94-106.

35. Vyas TK, Babbar AK, Sharma RK, Singh S, Misra A. Preliminary braintargeting studies on intranasal mucoadhesive microemulsions of sumatriptan. AAPS PharmSciTech. 2006;7:E8.

36. Mandal S, Mandal SD, Chuttani K, Dharamsi A, Subudhi BB. Transnasomucosal mucoadhesive microemulsion of zaltoprofen: a comparative brain distribution study. J Drug Deliv Sci Technol. 2017;39:237-246.

37. Chen $X$, Zhi F, Jia X, Zhang $X$, Ambardekar R, Meng Z, Paradkar AR, $\mathrm{Hu} Y$, Yang $Y$. Enhanced brain targeting of curcumin by intranasal administration of a thermosensitive poloxamer hydrogel. J Pharm Pharmacol. 2013;65:807-816.

38. Tian $X H$, Lin XN, Wei F, Feng W, Huang ZC, Wang P, Ren L, Diao Y. Enhanced brain targeting of temozolomide in polysorbate- 80 coated polybutylcyanoacrylate nanoparticles. Int J Nanomedicine. 2011;6:445452. 